\title{
Synthesis of $[1,2,3]$ triazolo[1,5-a][1,2,4]triazolo[5,1-c]pyrazines
}

\author{
Tomaz Trcek and Bojan Vercek* \\ Faculty of Chemistry and Chemical Technology, University of Ljubljana, Askerceva 5, 1000 \\ Ljubljana, Slovenia \\ E-mail: bojan.vercek@uni-lj.si
}

Dedicated to Professor Branko Stanovnik on his $65^{\text {th }}$ birthday

(received 30 Sep 03; accepted 15 Jan 04; published on the web 31 Jan 04)

\begin{abstract}
Some derivatives of the $[1,2,3]$ triazolo[ $[1,5-a][1,2,4]$ triazolo[5,1-c]pyrazine system have been synthesized using ethyl 4-amino-3-cyano[1,2,3]triazolo[1,5-a]pyrazine-6-carboxylate as the starting compound.
\end{abstract}

Keywords: $[1,2,3]$ Triazolo[1,5-a]pyrazine, $[1,2,3]$ triazolo[1,5-a][1,2,4]triazolo[5,1-c]pyrazine

\section{Introduction}

Diaminomaleonitrile (DAMN) and its $N$-substituted derivatives are very useful compounds in heterocyclic synthesis. ${ }^{1,2}$ Recently we designed novel approaches to $\beta$-(1,2,3-triazol-1-yl)- $\alpha$ amino acid derivatives, ${ }^{3} \beta$-(imidazol-1-yl)- $\alpha$-amino acid derivatives, ${ }^{4}$ imidazo[1,5-a]pyrazines, ${ }^{4}$ and $[1,2,3]$ triazolo[1,5-a]pyrazines ${ }^{5}$ based on the reaction of DAMN with 4-ethoxymethylidene2-phenyloxazol-5(4H)-one, ${ }^{6}$ (Scheme 1).

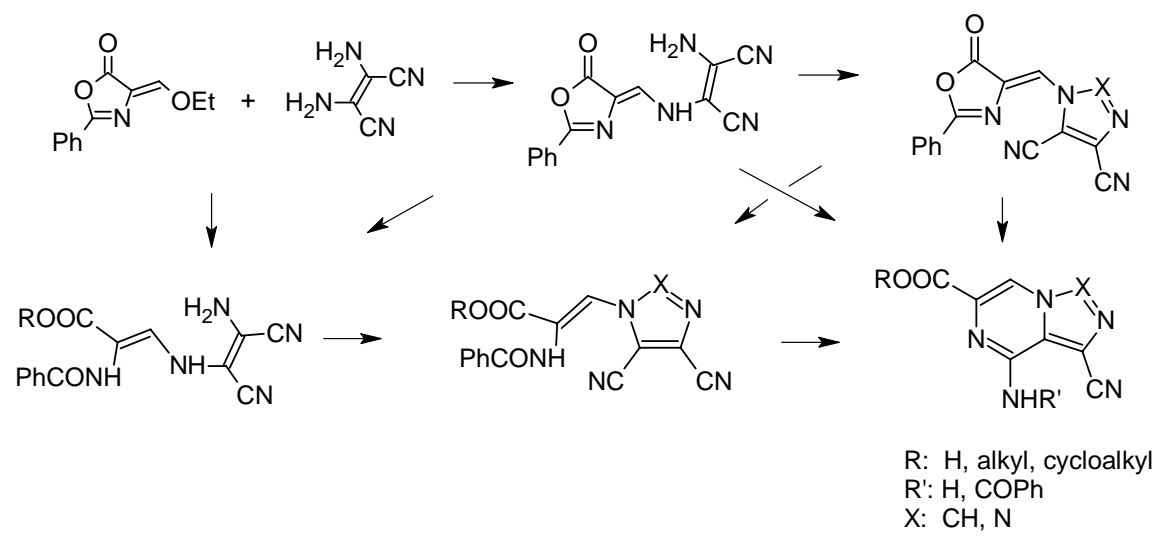

\section{Scheme 1}


As an extension of these investigations we describe here further functionalization of the $[1,2,3]$ triazolo[1,5-a]pyrazine system and the synthesis of some [1,2,3]triazolo[1,5-a][1,2,4]triazolo[5,1-c]pyrazines. We focused on the formation of 1,2,4-triazole ring fused to the $\mathrm{N}(5)=\mathrm{C}(4)\left(\mathrm{NH}_{2}\right)$ unit of the amine $1,{ }^{5}$ (Figure 1), by the general synthetic method that use $N, N$ dimethylformamide dimethyl acetal (DMFDMA), hydroxylamine hydrochloride, and polyphosphoric acid (PPA) as the main required reagents, ${ }^{7,8}$ thinking that this procedure could be extended to the heterocyclic system 1.

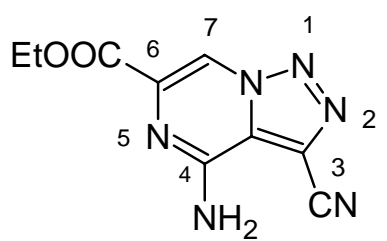

1

\section{Figure 1}

This transformation seemed to be of interest since it would represent the first entry to this tricyclic system. Besides, a competitive cyclization involving the amino and cyano groups might also be possible giving another tricyclic system. This type of reaction, but with the two groups in the same ring, had been observed in some heterocycles, for example in 2-amino-3-cyanopyridine. ${ }^{9,10}$ In addition, [1,2,3]triazoloazines with a bridgehead nitrogen atom exhibit valence tautomerism and could suffer lost of dinitrogen forming azine derivatives, via the corresponding diazomethylazines. ${ }^{11-14}$

\section{Results and Discussion}

Thus, reaction of the amine 1 with DMFDMA in refluxing chloroform afforded the expected amidine 2, which on subsequent treatment with excess hydroxylamine hydrochloride in presence of triethylamine gave compound 3. Heating 3 with PPA gave the tricyclic derivative 4. Treatment of $\mathbf{3}$ with acetic anhydride at room temperature resulted in the formation of the monoacetate 5, whereas reaction under reflux gave the diacetate $\mathbf{6}$. Attempts to form the 1,2,4triazole ring by another procedure, ${ }^{15}$ heating 5 in water failed, giving the amidoxime 7 as the main product, indicating that hydrolysis of the acetylated formamidoxime moiety took place. Compound 7 was also prepared by heating 1 with excess of hydroxylamine hydrochloride and triethylamine in ethanol. The amidoxime moiety of $\mathbf{4}$ was cyclized into an 1,2,4-oxadiazole ring. This conversion was carried out by treatment of $\mathbf{4}$ with acetic anhydride at room temperature to give the acetate $\mathbf{8}$, followed by subsequent cyclization to 9 by heating in water, (Scheme 2). 
The structures of all new compounds were elucidated on the basis of ${ }^{1} \mathrm{H},{ }^{13} \mathrm{C}$ and $2 \mathrm{D}$ (HMQC, HMBC) NMR spectroscopy and HRMS data. The chemical shifts attributed to the C-3, $\mathrm{C}-4, \mathrm{C}-6, \mathrm{CO}, \mathrm{CN}$, and $\mathrm{C}\left(\mathrm{NH}_{2}\right)=\mathrm{NO}$ carbons in the triazolopyrazine derivatives and to the $\mathrm{C}-2$, $\mathrm{C}-5, \mathrm{C}-10, \mathrm{C}-10 \mathrm{a}, \mathrm{C}-10 \mathrm{~b}, \mathrm{CO}$, and $\mathrm{C}(\mathrm{N})=\mathrm{NO}$ carbons in the tricyclic derivatives were distinguished on the basis of their HMBC connectivities. For example, the HMBC spectrum of 3 showed correlations between the $\mathrm{H}-7$ proton and the $\mathrm{C}-3 \mathrm{a}, \mathrm{C}-6$, and $\mathrm{CO}$ carbons, between the $\mathrm{NH}_{2}$ protons and the $\mathrm{C}-3$ carbon, between the $\mathrm{OH}$ proton (in the amidoxime moiety at the C-3 position) and the $\mathrm{C}-\mathrm{NH}_{2}$ carbon, between the $\mathrm{CH}$ proton and the $\mathrm{C}-4$ carbon, between the $\mathrm{NH}$ proton (in the amidoxime moiety at the C-4 position) and the C-3a, C-4 and C- 6 carbons, and between the $\mathrm{OCH}_{2}$ protons and the C-6 carbon.

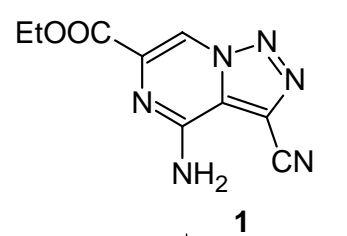

(ii)<smiles>CCOC(=O)c1cn2nnc(/C(N)=N\O)c2c(N)n1</smiles>

7

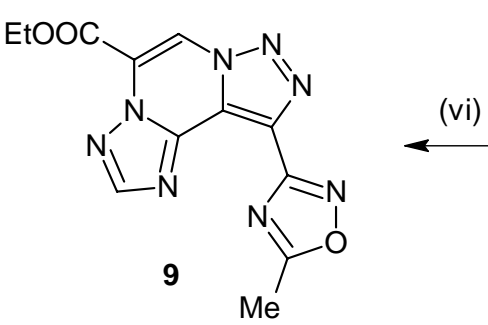

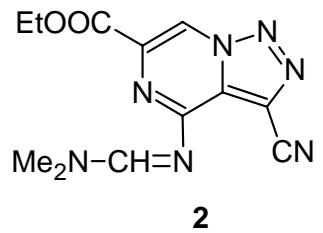

2

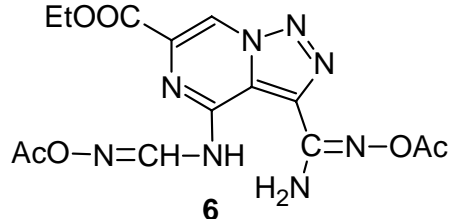

(ii)
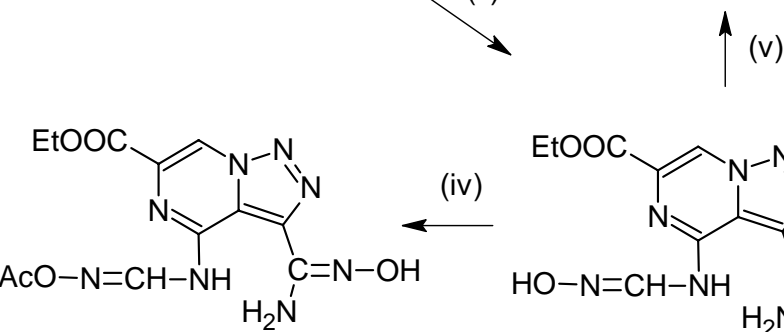

5

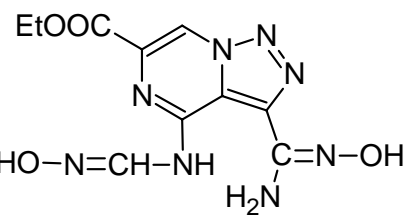

3

(iii)

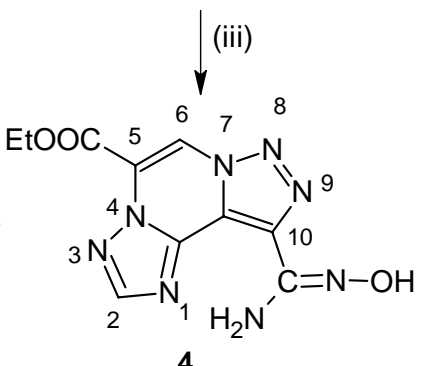

8

Scheme 2. Reagents and conditions: (i) DMFDMA, $\mathrm{CHCl}_{3}, \Delta$; (ii) $\mathrm{NH}_{2} \mathrm{OH} \cdot \mathrm{HCl}, \mathrm{Et}_{3} \mathrm{~N}$, ethanol, $\Delta$; (iii) PPA, $\Delta$; (iv) $\mathrm{Ac}_{2} \mathrm{O}$, rt; (v) $\mathrm{Ac}_{2} \mathrm{O}, \Delta$; (vi) $\mathrm{H}_{2} \mathrm{O}, \Delta$.

\section{Conclusions}

We have succeeded in the preparation of some derivatives of the $[1,2,3]$ triazolo[1,5-a][1,2,4]triazolo[5,1-c]pyrazine system. No reaction has been observed leading to the formation of a third 
ring by the participation of the amino and cyano groups or to the formation of pyrazine derivatives via $[1,2,3]$ triazolopyrazine-diazomethylpyrazine valence tautomerism.

\section{Experimental Section}

General Procedures. Melting points were determined on a Kofler micro hot stage and are uncorrected. IR spectra were recorded on a Bio-Rad FTS 3000MX spectrophotometer. NMR spectra were recorded on a Bruker AVANCE DPX-300 spectrometer (300 MHz for ${ }^{1} \mathrm{H}$ and 75.5 $\mathrm{MHz}$ for ${ }^{13} \mathrm{C}$ ) in $\mathrm{DMSO}-\mathrm{d}_{6}$ with TMS as an internal standard. The coupling constants $(J)$ are given in Hz. MS spectra were obtained on a VG-Analytical AutoSpec Q instrument. Compound 1 was prepared as described in the literature. ${ }^{5}$ Commercial compounds were used without purification as supplied by merchants.

Ethyl 3-cyano-4-(dimethylaminomethylideneamino)[1,2,3]triazolo[1,5-a]pyrazine-6-carboxylate (2). A mixture of $0.93 \mathrm{~g}(4 \mathrm{mmol})$ of $1,1.92 \mathrm{~g}(16 \mathrm{mmol})$ of DMFDMA, and $36 \mathrm{~mL}$ of chloroform was heated under reflux for $30 \mathrm{~min}$. The reaction mixture was concentrated to dryness in vacuo to give $1.14 \mathrm{~g}(99 \%)$ of 2 ; mp $218-219^{\circ} \mathrm{C}$ (ethanol). IR (KBr): 2244, 1734, 1707, 1630, 1599, 1496, 1452, 1419, $1396 \mathrm{~cm}^{-1} .{ }^{1} \mathrm{H}-\mathrm{NMR} \delta: 1.37$ (t, 3H, J=7.2, $\left.\mathrm{CH}_{2} \mathrm{CH}_{3}\right), 3.22$ $\left(\mathrm{s}, 3 \mathrm{H}, \mathrm{NCH}_{3}\right), 3.30$ (s, 3H, $\left.\mathrm{NCH}_{3}\right), 4.38$ (q, 2H, J=7.2, $\left.\mathrm{CH}_{2} \mathrm{CH}_{3}\right), 8.76(\mathrm{~s}, 1 \mathrm{H}, \mathrm{CH}), 9.26(\mathrm{~s}, 1 \mathrm{H}$, H-7). ${ }^{13} \mathrm{C}-\mathrm{NMR} \delta$ : $14.0\left(\mathrm{CH}_{2} \mathrm{CH}_{3}\right), 35.0\left(\mathrm{NCH}_{3}\right), 41.0\left(\mathrm{NCH}_{3}\right), 61.6\left(\mathrm{CH}_{2} \mathrm{CH}_{3}\right), 112.0(\mathrm{C}-3)$, 113.1 (CN), 116.2 (C-7), 129.4 (C-3a), 134.2 (C-6), 154.1 (C-4), 156.8 (CH), 163.1 (COOEt). MS (EI, m/z, \%): $287\left(\mathrm{M}^{+}, 16\right)$. HRMS Calc. for $\mathrm{C}_{12} \mathrm{H}_{13} \mathrm{~N}_{7} \mathrm{O}_{2}$ : 287.1131. Found: 287.1140.

Ethyl 3-(aminohydroxyiminomethyl)-4-(hydroxyiminomethylamino)[1,2,3]triazolo[1,5-a]pyrazine-6-carboxylate (3). A mixture of $2.30 \mathrm{~g}(8 \mathrm{mmol})$ of $2,1.68 \mathrm{~g}(24 \mathrm{mmol})$ of hydroxylamine hydrochloride, $2.42 \mathrm{~g}$ (24 mmol) of triethylamine, and $300 \mathrm{~mL}$ of ethanol was heated under reflux for $1 \mathrm{~h}$. Upon cooling to room temperature the separated solid was collected by filtration to give $1.60 \mathrm{~g}(65 \%)$ of $3 ; \mathrm{mp} 228-230^{\circ} \mathrm{C}$ (ethanol). IR (KBr): $3498,3424,3393$, $3312,1732,1660,1582,1562,1519,1439,1286,1224,1194,905,801 \mathrm{~cm}^{-1} .{ }^{1} \mathrm{H}-\mathrm{NMR} \delta: 1.35(\mathrm{t}$, $\left.3 \mathrm{H}, J=7.1, \mathrm{CH}_{2} \mathrm{CH}_{3}\right), 4.36$ (q, 2H, J=7.2, $\left.\mathrm{CH}_{2} \mathrm{CH}_{3}\right), 6.50$ (s, 2H, $\mathrm{NH}_{2}$ ), 7.79 (d, $1 \mathrm{H}, J=9.2$, NH$\mathrm{CH}=), 9.12$ (s, 1H, H-7), $9.82\left(\mathrm{~s}, 1 \mathrm{H}, \mathrm{C}(=\mathrm{NOH}) \mathrm{NH}_{2}\right), 10.66(\mathrm{~s}, 1 \mathrm{H}, \mathrm{NH}-\mathrm{CH}=\mathrm{NOH}), 12.69$ (d, $1 \mathrm{H}, J=9.4, \mathrm{NH}-\mathrm{CH}=) .{ }^{13} \mathrm{C}-\mathrm{NMR} \delta: 14.0\left(\mathrm{CH}_{2} \mathrm{CH}_{3}\right), 61.5\left(\mathrm{CH}_{2} \mathrm{CH}_{3}\right), 115,7(\mathrm{C}-7), 119.4(\mathrm{C}-3 \mathrm{a})$, 132.4 (C-6), 132.8 (C-3), $133.2(\mathrm{NH}-\mathrm{CH}=\mathrm{NOH}), 145.0(\mathrm{C}-4), 146.3\left(\mathrm{C}(=\mathrm{NOH}) \mathrm{NH}_{2}\right), 163.1$ (COOEt). MS (EI, m/z, \%): $308\left(\mathrm{M}^{+}, 100\right)$. HRMS Calc. for $\mathrm{C}_{10} \mathrm{H}_{12} \mathrm{~N}_{8} \mathrm{O}_{4}$ : 308.0982. Found: 308.0991 .

Ethyl 10-(aminohydroxyiminomethyl)[1,2,3]triazolo[1,5-a][1,2,4]triazolo[5,1-c]pyrazine-5carboxylate (4). A mixture of $308 \mathrm{mg}(1 \mathrm{mmol})$ of 3 and $1 \mathrm{~g}$ of PPA was heated on an oil bath at $60^{\circ} \mathrm{C}$ for $24 \mathrm{~h}$. Upon cooling to room temperature the reaction mixture was diluted with $5 \mathrm{~mL}$ of water and neutralized with $\mathrm{NaHCO}_{3}$. The separated solid was collected by filtration and washed three times with $1 \mathrm{~mL}$ of water to give $210 \mathrm{mg}(72 \%)$ of 4 ; mp $177-179^{\circ} \mathrm{C}$ (ethanol). $\mathrm{IR}(\mathrm{KBr})$ : 
$3472,3377,3323,3202,3076,1743,1644,1470,1285,1269,1250,1227,1092,944,788 \mathrm{~cm}^{-1}$. ${ }^{1} \mathrm{H}-\mathrm{NMR} \delta: 1.41$ (t, 3H, J=7.0, $\left.\mathrm{CH}_{2} \mathrm{CH}_{3}\right), 4.48$ (q, 2H, J=7.2, $\left.\mathrm{CH}_{2} \mathrm{CH}_{3}\right), 6.56$ (s, 2H, $\mathrm{NH}_{2}$ ), 8.78 (s, $1 \mathrm{H}, \mathrm{H}-2), 9.56(\mathrm{~s}, 1 \mathrm{H}, \mathrm{H}-6), 10.14(\mathrm{~s}, 1 \mathrm{H}, \mathrm{OH}) .{ }^{13} \mathrm{C}-\mathrm{NMR} \delta: 13.9\left(\mathrm{CH}_{2} \mathrm{CH}_{3}\right), 62.7\left(\mathrm{CH}_{2} \mathrm{CH}_{3}\right)$, 118.6 (C-5), 119.2 (C-6), 121.6 (C-10a), 135.3 (C-10), 143.2 (C-10b), 143.5 (C=NOH), 152.8 (C-2), 158.1 (COOEt). MS (EI, m/z, \%): $290\left(\mathrm{M}^{+}, 27 \%\right)$. HRMS Calc. for $\mathrm{C}_{10} \mathrm{H}_{10} \mathrm{~N}_{8} \mathrm{O}_{3}$ : 290.0876. Found: 290.0883 .

Ethyl 4-(acetyloxyiminomethylamino)-3-(aminohydroxyiminomethyl)[1,2,3]triazolo[1,5-a]pyrazine-6-carboxylate (5). A mixture of $308 \mathrm{mg}(1 \mathrm{mmol})$ of 3 and $3 \mathrm{~mL}$ of acetic anhydride was stirred at room temperature for $12 \mathrm{~h}$. The a solid was separated, collected by filtration and washed with $2 \mathrm{~mL}$ of ethanol to give $291 \mathrm{mg}(83 \%)$ of 5 ; mp $180-182^{\circ} \mathrm{C}$ (ethanol). IR ( $\mathrm{KBr}$ ): 3506, 3391, 1741, 1651, 1528, 1443, 1285, 1234, 1214, 1188, 1073, 953, 869, $675 \mathrm{~cm}^{-1} .{ }^{1} \mathrm{H}-$ NMR $\delta: 1.37$ (t, 3H, J=7.1, $\left.\mathrm{CH}_{2} \mathrm{CH}_{3}\right), 2.28\left(\mathrm{~s}, 3 \mathrm{H}, \mathrm{OCOCH}_{3}\right), 4.38$ (q, $\left.2 \mathrm{H}, J=7.0, \mathrm{CH}_{2} \mathrm{CH}_{3}\right), 6.79$ (s, 2H, $\left.\mathrm{NH}_{2}\right), 8.38(\mathrm{~d}, 1 \mathrm{H}, J=9.6, \mathrm{NH}-\mathrm{CH}=), 9.29(\mathrm{~s}, 1 \mathrm{H}, \mathrm{H}-7), 9.72(\mathrm{~s}, 1 \mathrm{H}, \mathrm{OH}), 13.34(\mathrm{~d}, 1 \mathrm{H}$, $J=9.6, \mathrm{NH}-\mathrm{CH}=) .{ }^{13} \mathrm{C}-\mathrm{NMR} \delta: 14.0\left(\mathrm{CH}_{2} \mathrm{CH}_{3}\right), 19.6\left(\mathrm{OCOCH}_{3}\right), 61.6\left(\mathrm{CH}_{2} \mathrm{CH}_{3}\right), 117.2(\mathrm{C}-7)$, 119.8 (C-3a), $132.0(\mathrm{C}-6), 132.6(\mathrm{C}-3), 138.8(\mathrm{NH}-\mathrm{CH}=\mathrm{N}), 144.6(\mathrm{C}-4), 147.6\left(\mathrm{C}(=\mathrm{NOH}) \mathrm{NH}_{2}\right)$, 162.7 (COOEt), $168.3\left(\mathrm{OCOCH}_{3}\right)$. MS (EI, $\left.\mathrm{m} / \mathrm{z}, \%\right): 350\left(\mathrm{M}^{+}, 47\right)$. HRMS Calc. for $\mathrm{C}_{12} \mathrm{H}_{14} \mathrm{~N}_{8} \mathrm{O}_{5}$ : 350.1087. Found: 350.1097.

Ethyl 3-(acetyloxyiminoaminomethyl)-4-(acetyloxyiminomethylamino)[1,2,3]triazolo[1,5-a]pyrazine-6-carboxylate (6). A mixture of $925 \mathrm{mg}(3 \mathrm{mmol})$ of 3 and $9 \mathrm{~mL}$ of acetic anhydride was heated with occasionally stirring on an oil bath at $95^{\circ} \mathrm{C}$ for $8 \mathrm{~h}$. Upon cooling to room temperature the separated solid was collected by filtration and washed with $3 \mathrm{~mL}$ of ethanol to give $790 \mathrm{mg}(67 \%)$ of $\mathbf{6}$; $\mathrm{mp} 180-181^{\circ} \mathrm{C}$ (ethanol). IR (KBr): 3455, 3352, 1775, 1751, 1725, 1638, 1523, 1364, 1271, 1206, 1173, 1007, 941, $877 \mathrm{~cm}^{-1} .{ }^{1} \mathrm{H}-\mathrm{NMR} \delta: 1.37$ (t, 3H, J=7.0, $\left.\mathrm{CH}_{2} \mathrm{CH}_{3}\right), 2.16\left(\mathrm{~s}, 3 \mathrm{H}, \mathrm{OCOCH}_{3}\right), 2.20\left(\mathrm{~s}, 3 \mathrm{H}, \mathrm{OCOCH}_{3}\right), 4.40$ (q, $\left.2 \mathrm{H}, J=7.0, \mathrm{CH}_{2} \mathrm{CH}_{3}\right), 7.80(\mathrm{~s}$, $\left.2 \mathrm{H}, \mathrm{NH}_{2}\right), 8.38(\mathrm{~d}, 1 \mathrm{H}, J=9.8$, NH-CH=), $9.40(\mathrm{~s}, 1 \mathrm{H}, \mathrm{H}-7), 12.81$ (d, $1 \mathrm{H}, J=9.8, \mathrm{NH}-\mathrm{CH}=) .{ }^{13} \mathrm{C}-$ NMR $\delta: 14.0\left(\mathrm{CH}_{2} \mathrm{CH}_{3}\right), 19.3\left(\mathrm{OCOCH}_{3}\right), 19.6\left(\mathrm{OCOCH}_{3}\right), 61.7\left(\mathrm{CH}_{2} \mathrm{CH}_{3}\right), 117.5(\mathrm{C}-7), 120.5$ (C-3a), 130.4 (C-3), 132.0 (C-6), $138.3(\mathrm{NH}-\mathrm{CH}=\mathrm{N}), 144.2(\mathrm{C}-4), 152.3\left(\mathrm{C}-\mathrm{NH}_{2}\right), 162.6$ (COOEt), $167.9\left(\mathrm{OCOCH}_{3}\right), 168.1\left(\mathrm{OCOCH}_{3}\right)$. MS (EI, m/z, \%): $392\left(\mathrm{M}^{+}, 65\right)$. HRMS Calc. for $\mathrm{C}_{14} \mathrm{H}_{16} \mathrm{~N}_{8} \mathrm{O}_{6}$ : 392.1193. Found: 392.1204.

Ethyl 4-amino-3-(aminohydroxyiminomethyl)[1,2,3]triazolo[1,5-a]pyrazine-6-carboxylate (7).

a) A mixture of $232 \mathrm{mg}(1 \mathrm{mmol})$ of $1,210 \mathrm{mg}(3 \mathrm{mmol})$ of hydroxylamine hydrochloride, 303 ( $3 \mathrm{mmol}$ ) of triethylamine, and $20 \mathrm{~mL}$ of ethanol was heated under reflux for $1 \mathrm{~h}$. Upon cooling to room temperature the separated solid was collected by filtration to give $208 \mathrm{mg}(78 \%)$ of 7 ; $\mathrm{mp} 290-291^{\circ} \mathrm{C}$ (ethanol). IR (KBr): 3501, 3393, 3118, 1734, 1659, 1618, 1572, 1527, 1422, 1304, 1273, 1221, 1093, 1062, $966 \mathrm{~cm}^{-1} .{ }^{1} \mathrm{H}-\mathrm{NMR} \delta: 1.33$ (t, 3H, J=7.2, $\left.\mathrm{CH}_{2} \mathrm{CH}_{3}\right), 4.33$ (q, 2H, $\left.J=7.2, \mathrm{CH}_{2} \mathrm{CH}_{3}\right), 6.33$ (s, $\left.2 \mathrm{H}, \mathrm{C}(=\mathrm{NOH}) \mathrm{NH}_{2}\right), 8.37$ (s, $\left.1 \mathrm{H}, \mathrm{NH}_{2}\right), 8.79$ (s, $\left.1 \mathrm{H}, \mathrm{H}-7\right), 9.62(\mathrm{~s}, 1 \mathrm{H}$,

$\left.\mathrm{NH}_{2}\right), 10.19$ (s, $\left.1 \mathrm{H}, \mathrm{OH}\right) .{ }^{13} \mathrm{C}-\mathrm{NMR} \delta: 14.1\left(\mathrm{CH}_{2} \mathrm{CH}_{3}\right), 61.2\left(\mathrm{CH}_{2} \mathrm{CH}_{3}\right), 111.9(\mathrm{C}-7), 119.0(\mathrm{C}-3$ a), 132.9 (C-3), 134.0 (C-6), 145.8 (C=NOH), 151.5 (C-4), 163.8 (COOEt). MS (EI, m/z, \%): $265\left(\mathrm{M}^{+}, 20 \%\right)$. HRMS Calc. for $\mathrm{C}_{9} \mathrm{H}_{11} \mathrm{~N}_{7} \mathrm{O}_{3}$ : 265.0923. Found: 265.0933. 
b) A suspension of $350 \mathrm{mg}$ of 5 ( $1 \mathrm{mmol})$ and $32 \mathrm{~mL}$ of water was heated under reflux for $11 \mathrm{~h}$. Upon cooling to room temperature the separated solid was collected by filtration to give $160 \mathrm{mg}$ of a crude mixture of 7 and 3 in a ratio of 10:1, estimated on the basis of ${ }^{1} \mathrm{H}$ NMR spectroscopy.

Ethyl 10-(acetyloxyiminoaminomethyl)[1,2,3]triazolo[1,5-a][1,2,4]triazolo[5,1-c]pyrazine-5carboxylate (8). A mixture of $871 \mathrm{mg}(3 \mathrm{mmol})$ of 5 and $3 \mathrm{~mL}$ of acetic anhydride was stirred at room temperature for $12 \mathrm{~h}$. The separated solid was collected by filtration and washed with $2 \mathrm{~mL}$ of ethanol to give $855 \mathrm{mg}(86 \%)$ of $\mathbf{8} ; \mathrm{mp} 169-170^{\circ} \mathrm{C}$ (ethanol). IR (KBr): 3403, 3200, 3080, $1755,1733,1717,1647,1386,1360,1339,1288,1263,1249,1229,1096,1011,945 \mathrm{~cm}^{-1} .{ }^{1} \mathrm{H}-$ NMR $\delta: 1.41\left(\mathrm{t}, 3 \mathrm{H}, J=7.2, \mathrm{CH}_{2} \mathrm{CH}_{3}\right), 2.24\left(\mathrm{~s}, 3 \mathrm{H}, \mathrm{COCH}_{3}\right), 4.49$ (q, 2H, J=7.0, $\left.\mathrm{CH}_{2} \mathrm{CH}_{3}\right), 7.51$ (s, 2H, $\left.\mathrm{NH}_{2}\right), 8.84$ (s, 1H, H-2), 9.66 (s, $\left.\left.1 \mathrm{H}, \mathrm{H}-6\right) .{ }^{13} \mathrm{C}-\mathrm{NMR} \delta: 13.9 \mathrm{CH}_{2} \mathrm{CH}_{3}\right), 19.8\left(\mathrm{CH}_{3}\right), 62.8$ $\left(\mathrm{CH}_{2} \mathrm{CH}_{3}\right), 119.0$ (C-5), 119.2 (C-6), 123.4 (C-10a), 133.2 (C-10), 142.8 (C-10b), 148.7 (C$\mathrm{NH}_{2}$ ), 153.0 (C-2), 158.0 (COOEt), $168.6\left(\mathrm{COCH}_{3}\right)$. MS (EI, m/z, \%): $332\left(\mathrm{M}^{+}, 4 \%\right)$. HRMS Calc. for $\mathrm{C}_{12} \mathrm{H}_{12} \mathrm{~N}_{8} \mathrm{O}_{4}$ : 332.0982. Found: 332.0991 .

Ethyl 10-(5-methyl-1,2,4-oxadiazol-3-yl)[1,2,3]triazolo[1,5-a][1,2,4]triazolo[5,1-c]pyrazine5-carboxylate (9). A mixture of $332 \mathrm{mg}(1 \mathrm{mmol})$ of 8 and $8 \mathrm{~mL}$ of water was heated under reflux for $6 \mathrm{~h}$. Upon cooling to room temperature the separated solid was collected by filtration to give $180 \mathrm{mg}(57 \%)$ of $\mathbf{9} ; \mathrm{mp} 228-230^{\circ} \mathrm{C}$ (ethanol). IR (KBr): $3096,1746,1578,1428,1383$, 1303, 1278, 1262, 1233, 1172, 1092, 1020, 950, $738 \mathrm{~cm}^{-1} .{ }^{1} \mathrm{H}-\mathrm{NMR} \delta: 1.42$ (t, 3H, J=7.0, $\mathrm{CH}_{2} \mathrm{CH}_{3}$ ), 2.77 (s, 3H, $\mathrm{CH}_{3}$ ), 4.50 (q, 2H, J=7.0, $\left.\mathrm{CH}_{2} \mathrm{CH}_{3}\right), 8.79$ (s, 1H, H-2), 9.67 (s, 1H, H-6). $\left.{ }^{13} \mathrm{C}-\mathrm{NMR} \delta: 12.0\left(\mathrm{CH}_{3}\right), 13.9 \mathrm{CH}_{2} \mathrm{CH}_{3}\right), 62.8\left(\mathrm{CH}_{2} \mathrm{CH}_{3}\right), 118.8(\mathrm{C}-6), 119.6(\mathrm{C}-5), 124.4(\mathrm{C}-$ 10a), 128.6 (C-10), 142.6 (C-10b), 153.8 (C-2), 158.0 (COOEt), 161.0 (C-3'), 177.9 (C-5'). MS (EI, $m / z, \%): 314\left(\mathrm{M}^{+}, 7 \%\right)$. HRMS Calc. for $\mathrm{C}_{12} \mathrm{H}_{10} \mathrm{~N}_{8} \mathrm{O}_{3}: 314.0876$. Found: 314.0885 .

\section{Acknowledgements}

We thank The Ministry of Education, Science and Sport of Slovenia (P0-0503-0103) for financial support.

\section{References}

1. Erian, A. W. Chem. Rev. 1993, 93, 1991.

2. Al-Azmi, A.; Elassar, A.-Z. A.; Booth, B. L. Tetrahedron 2003, 59, 2749.

3. Polak, M.; Vercek, B. Synth. Commun. 2000, 30, 2863.

4. Trcek, T.; Meden, A.; Vercek, B. Synlett 2000, 1458.

5. Jug, T.; Polak, M.; Trcek, T.; Vercek, B. Heterocycles 2002, 56, 353.

6. Cornforth, J. W. In The Chemistry of Penicillin; Clarke, H. T.; Johnson, J. R.; Robinson, R.; Eds., Princeton University Press: Princeton, 1949; p 803.

7. Polanc, S.; Vercek, B.; Stanovnik, B.; Tisler, M. Tetrahedron Lett. 1973, 1677. 
8. Polanc, S.; Vercek, B.; Sek, B.; Stanovnik, B.; Tisler, M. J. Org. Chem. 1974, 39, 2143.

9. Vercek, B.; Leban, I.; Stanovnik, B.; Tisler, M. Heterocycles 1978, 9, 1327.

10. Vercek, B.; Leban, I.; Stanovnik, B.; Tisler, M. J. Org. Chem. 1979, 44, 1695.

11. Sutherland, D. R.; Tennant, G. J. Chem. Soc. Chem. Commun. 1969, 1070.

12. Sutherland, D. R.; Tennant, G. J. Chem. Soc. (C) 1971, 2156.

13. Novinson, T.; Dea, P.; Okabe, T. J. Org. Chem. 1976, 41, 385.

14. Wentrup, C. Helv. Chim. Acta 1978, 61, 1755.

15. Vercek, B.; Stanovnik, B.; Tisler, M.; Zrimsek, Z. Org. Prep. Proc. Int. 1978, 293. 